\title{
Cosyntropin as a diagnostic agent in the screening of patients for adrenocortical insufficiency
}

This article was published in the following Dove Press journal:

Clinical Pharmacology:Advances and Applications

24 April 2010

Number of times this article has been viewed

\section{David D Hamilton \\ Bryan A Cotton}

Department of Surgery, The University of Texas Health Science

Center, Houston, TX, USA
Correspondence: Bryan A Cotton The University of Texas Health Science Center at Houston, Department of Surgery and The Center for Translational Injury Research, Houston, TX, USA

Tel +I 7135007354

Fax +17135127135

Email bryan.a.cotton@uth.tmc.edu
Abstract: Adrenocortical insufficiency occurs when there is inadequate release of cortisol from the adrenal cortex. Disturbances of the hypothalamic-pituitary-adrenal axis are common following trauma, surgical stress, and critical illness. While this is often a protective mechanism, these responses may become "uncoupled" or maladaptive resulting in an exacerbation of organ failure and higher mortality rates. In these clinical settings, the patient presents with a persistent systemic inflammation state, a hyperdynamic cardiovascular response, and vasopressor dependent shock. As such, the occurrence of adrenal insufficiency in the setting of critical illness is most appropriately termed critical illness-related corticosteroid insufficiency. In these settings, recent data suggests that these patients may benefit from a short course of low-dose steroid replacement therapy. Cosyntropin, a synthetic derivative of adrenocorticotropic hormone, is being used with increased frequency in the evaluation and diagnosis of adrenocortical insufficiency in this patient population. A random cortisol level is checked before a $250-\mu \mathrm{g}$ injection of cosyntropin and then 30-60 minutes later. The cortisol levels and response to cosyntropin may be interpreted to identify an insufficient adrenal response. Of note, the setting of critical illness can greatly affect the cosyntropin test sensitivity on identifying adrenal insufficiency. Changes in the stress response during critical illness combined with the resuscitation and management of these patients greatly disturbs serum protein levels, especially those of albumin and transcortin. Common intensive care unit (ICU) diagnoses such as sepsis and malnutrition can increase baseline levels and blunt the cortisol response to cosyntropin stimulation, respectively. As well, numerous pharmacological agents routinely used in the ICU have been shown to interfere with cortisol levels and cosyntropin responsiveness. While steroids have a place in the ICU, specific dosing and length of administration remain inconsistent

Keywords: critical illness, ACTH, cosyntropin, adrenal insufficiency

\section{Adrenocortical insufficiency}

Adrenocortical insufficiency occurs when there is inadequate release of cortisol from the adrenal cortex. The incidence rate of adrenal insufficiency is approximately five cases per million people per year with a prevalence of 35 to 60 cases per million in Western countries. ${ }^{1,2}$ Primary adrenal insufficiency is due to an impairment of the adrenal glands, while secondary adrenal insufficiency is caused by impairment of the pituitary gland, tertiary insufficiency is due to hypothalamic dysfunction. Intrinsic adrenal disease arises from three general mechanisms: congenital adrenal hypoplasia, defective steroid synthesis, and adrenal destruction (from autoimmune causes, infection, adrenal replacement by metastatic tumor, and adrenal hemorrhage as in Waterhouse-Friderichsen syndrome) (Table 1). 
Table I Causes of adrenal insufficiency

\begin{tabular}{ll}
\hline Primary & Autoimmune adrenalitis \\
& Metastatic carcinoma \\
Infection (HIV,Tb, Fungal) & Hemorrhage/Infarction (disseminated intravascular \\
coagulation, meningococcemia, anticoagulation, & heparin-induced thrombocytopenia) \\
& Sepsis \\
& Drugs (ketoconazole, etomidate, mitotane, \\
& metyrapone) \\
& Hypothermia \\
& Genetic (adrenoleukodystrophy, autoimmune \\
& polyglandular disease) \\
& latrogenic (post-adrenalectomy) \\
& Chronic steroid use \\
& Pituitary or metastatic tumor \\
& Pituitary surgery or radiation \\
Secondary & Empty-sella syndrome \\
& Craniopharyngioma \\
Infection (HIV, sarcoidosis, histiocytosis) \\
Head trauma \\
Post-partum pituitary necrosis (Sheehan's syndrome)
\end{tabular}

Abbreviations: HIV, human immunodeficiency virus; Tb, tuberculosis.

Secondary adrenal insufficiency appears mainly as a manifestation of discontinuation of steroid therapy. Less common causes of secondary adrenal insufficiency include pan-hypopituitarism secondary to neoplastic or infiltrative replacement, granulomatous disease, and pituitary hemorrhage or infarction (Sheehan's syndrome). The classical Addison's disease describes patients with tuberculous destruction of the adrenal gland manifested as anemic patients with skin hyperpigmentation, weakness, electrolyte disturbances, nausea, vomiting, and hypotension. Irritability, depression, and salt-craving are other chronic symptoms. While tuberculosis remains the most common cause of primary adrenal insufficiency in developing nations, autoimmune destruction of the adrenal glands has become the leading cause of primary adrenal insufficiency in Western countries. ${ }^{3}$

Acute adrenal insufficiency (Addisonian crisis) refers to the acute onset of hypotension, hyponatremia, hypoglycemia, hyperkalemia, and shock. Hyponatremia is present in approximately $90 \%$ of cases and hyperkalemia in $65 \%$. Additionally the blood urea nitrogen concentration is usually elevated. Hyperkalemia occurs because of aldosterone deficiency, and therefore is more common in patients with primary adrenal insufficiency than in patients with secondary adrenal insufficiency. Hypercalcemia occurs in $6 \%$ of all cases, and may be particularly marked in patients with coexisting thyrotoxicosis. However, free thyroxine concentrations are usually low or normal, and thyroid-stimulating hormone (TSH) values are frequently moderately elevated. ${ }^{4,5}$ Patients taking supraphysiologic dosages of exogenous steroids for more than three weeks are at risk for secondary adrenal insufficiency as return of a competent hypothalamic-pituitary-adrenal axis may take up to eight to twelve months. ${ }^{6}$

Disturbances of the hypothalamic-pituitary-adrenal axis (HPA) axis are common following trauma, surgical stress, and critical illness. ${ }^{6-11}$ This is often an adaptive and protective mechanism, the integrity of which, determines in part the response of the patient to injury and stress. However, the response to these challenges may become "uncoupled" or maladaptive, resulting in a systemic inflammatory response state that precipitates the development of multisystem organ failure and leads to higher mortality rates. Although "classic" adrenal insufficiency is uncommon in these settings, occult or relative adrenal insufficiency has been noted in up to $60 \%$ of severely ill or injured patients. ${ }^{12-14}$ In these clinical settings, the patient presents with a persistent systemic inflammation state, a hyperdynamic cardiovascular response, and vasopressor dependent shock. As such, the occurrence of adrenal insufficiency in the setting of critical illness is most appropriately termed critical illness-related corticosteroid insufficiency (CIRCI). ${ }^{28,44}$

Treatment of adrenal insufficiency centers around cortisol replacement. Over $90 \%$ of circulating cortisol is bound to corticosteroid-binding globulin with less than $10 \%$ in the free, biologically active form. ${ }^{15,16}$ Nonstressed daily production of cortisol in adults is approximately 15 to $25 \mathrm{mg} /$ day and the maximal stressed daily production of cortisol is approximately 200 to $350 \mathrm{mg} /$ day. Based on this data, a daily dose of hydrocortisone (or equivalent) of 25 to $200 \mathrm{mg} /$ day should be considered a low dose, 200 to $350 \mathrm{mg}$ /day should be considered as a physiological stress dose, 351 to $1,000 \mathrm{mg} /$ day is a supraphysiologic dose, and more than $1,000 \mathrm{mg} /$ day is considered as being high dose corticosteroid therapy. ${ }^{17}$ In the setting of relative or occult adrenal insufficiency, recent data suggests that these patients may benefit from a short course of low-dose steroid replacement therapy. This is usually delivered as $200 \mathrm{mg}$ of hydrocortisone in a divided dose.

\section{Cosyntropin: pharmacology and pharmacokinetics}

Cosyntropin is a synthetic derivative of adrenocorticotropic hormone (ACTH) that is used in the evaluation and diagnosis of patients with adrenocortical insufficiency. ACTH is a naturally occurring peptide hormone that is secreted by the anterior pituitary to act on the cells of the adrenal cortex to 
increase secretion of glucocorticoids, mineralocorticoids, and sex steroids. Cosyntropin is an open chain polypeptide containing the first 24 of the 39 amino acids of natural ACTH. It stimulates adrenal activity to the same extent of natural ACTH. ${ }^{18}$ The biologic activity of ACTH resides in the N-terminal portion of the molecule and the 1-20 amino acid residue is the minimal sequence retaining full activity. Progressive shortening of the chain beyond 20 amino acid residues results in partial or complete loss of activity. For example, the decrement from 20 to 19 results in a $70 \%$ loss of potency. Cosyntropin is sometimes referred to outside of the United States as tetracosactide.

The pharmacologic profile of cosyntropin is similar to that of purified natural ACTH. A single dose of $0.25 \mathrm{mg}$ of cosyntropin will stimulate the adrenal cortex maximally and to the same extent as 25 units of natural ACTH. The half-life of cortisol is in the range of 70 to 120 minutes. However, the half-life for cosyntropin is only 15 minutes. Administration is by intravenous or intramuscular injection and a rise in cortisol should generally be seen around 30 minutes after administration. Plasma cortisol levels usually peak about 45 to 60 minutes after injection and a normal response is an approximate doubling of the basal plasma cortisol value. A number of other agents may interfere with cosyntropin function and subsequent response. These include metyrapone, etomidate, ketoconazole, megesterol, and mitotate to name a few. In addition, agents such as rifampin and phenytoin may increase cortisol metabolism.

Cosyntropin has also been used to eliminate fluctuations in aldosterone levels during adrenal venous sampling for aldosteronoma localization. Blood is obtained from the right and left adrenal veins, the inferior vena cava, or the peripheral veins. The key diagnostic feature in the case of an adenoma is unilateral elevation of aldosterone, with suppression of contralateral aldosterone. Levels drawn from the inferior vena cava or peripheral samples should be higher than from the contralateral suppressed gland. ${ }^{19}$

Side effects of cosyntropin include nausea, anxiety, sweating, dizziness, itchy skin, redness and or swelling at injection site, palpitations, and facial flushing. ${ }^{18}$ Rarely seen side effects include fainting, headache, blurred vision, severe swelling, severe dizziness, trouble breathing, or an irregular heartbeat. A rare hypersensitivity reaction usually associated with a preexisting allergic disease or prior ACTH allergy is possible. Bradycardia, tachycardia, hypertension, peripheral edema have all been reported after administration of cosyntropin. Additionally, cosyntropin may accentuate the electrolyte loss associated with diuretic therapy. Cosyntropin is pregnancy Category $\mathrm{C}$; meaning it is not known if cosyntropin can cause
Table 2 American College of Critical Care Medicine's task force guidelines for diagnosis and management of adrenal insufficiency in critical illness

Diagnosis of adrenal insufficiency

I.Adrenal insufficiency is best diagnosed by a change in cortisol (after $250 \mu \mathrm{g}$ cosyntropin) of $<9 \mu \mathrm{g} / \mathrm{dL}$ or a random total cortisol of $<10 \mu \mathrm{g} / \mathrm{dL}$.

2. Free cortisol measurements cannot be recommended for routine use at this time.

3.ACTH stimulation testing should not be used to identify those patients with septic shock or ARDS who should receive glucocorticoid therapy.

\section{Treatment of adrenal insufficiency}

I. Hydrocortisone should be considered in patients with septic shock who have responded poorly to fluid resuscitation and vasopressor agents.

2. Moderate-dose glucocorticoid therapy should be considered in patients with early severe ARDS $\left(\mathrm{PaO}_{2} / \mathrm{FiO}_{2}\right.$ of $\left.<200\right)$ and before day 14 in patients with unresolving ARDS.

3. Patients with septic shock should be treated for seven days before tapering, while patients with early ARDS should be treated for 14 days before tapering.

Notes: Adapted from: Marik PE, Pastores SM, Annane D, et al. Recommendations for the diagnosis and management of corticosteroid insufficiency in critically ill adult patients: consensus statements from an international task force by the American College of Critical Care Medicine. Crit Care Med. 2008;36(6): 1937-1949.

Abbreviations: ACTH, adrenocorticotropic hormone; ARDS, acute respiratory distress syndrome.

fetal harm or affect reproduction capacity. Administration to a pregnant woman should only be if clearly needed. Caution should also be exercised when cosyntropin is administered to a woman who is breastfeeding. The extra-adrenal effects, which natural ACTH and cosyntropin have in common, include increased melanotropic activity, increased growth hormone secretion, and lipogenesis. These are considered to be without physiological or clinical significance.

\section{Cosyntropin and the diagnosis of adrenal insufficiency}

In the setting of primary and secondary adrenal insufficiency, random cortisol levels alone are usually not adequate in providing a diagnosis because of the wide fluctuations due to the pulsatile nature and diurnal variation of secretion. Cortisol levels typically are highest early in the morning and at their lowest near midnight. The cortisol response to cosyntropin displays great variability among healthy persons. Several factors affect the response including, stress level, body composition, serum corticosteroid-binding globulin level, and individual differences in the HPA axis. The cosyntropin test works well in patients with primary adrenal insufficiency, but the lower sensitivity in patients with secondary adrenal insufficiency necessitates the use of tests involving stimulation of the hypothalamus if the 
pretest probability is sufficiently high. The operating characteristics of the $250 \mu \mathrm{g}$ and $1 \mu \mathrm{g}$ cosyntropin tests are similar. ${ }^{20}$

The low-dose ACTH stimulation test $(1 \mu \mathrm{g})$ has been shown to be more sensitive and specific than the high-dose test $(250 \mu \mathrm{g})$, however; the high-dose test is preferred since the low-dose test has not been validated. ${ }^{20,21}$ The test has a reported specificity of $95 \%$, with sensitivities of $97 \%, 57 \%$, and $61 \%$ for primary adrenal insufficiency ( $250 \mu \mathrm{g}$ cosyntropin test), secondary adrenal insufficiency (250 $\mu \mathrm{g}$ cosyntropin test), and secondary adrenal insufficiency ( $1 \mu \mathrm{g}$ cosyntropin test), respectively. Therefore the cosyntropin stimulation test is helpful for ruling in secondary AI, but not for ruling it out. ${ }^{20}$ In unstressed subjects, adrenal insufficiency is confirmed when the baseline cortisol is less than $3 \mu \mathrm{g} / \mathrm{dL}$ or the $250 \mu \mathrm{g}$ ACTHstimulated cortisol is less than $18-20 \mu \mathrm{g} / \mathrm{dL} .{ }^{3,20,22}$ Alternatively suspected AI can be diagnosed with a post-cosyntropin level of at least $20 \mu \mathrm{g} / \mathrm{dL}$ for primary AI and at least $25-30 \mu \mathrm{g} / \mathrm{dL}$ for secondary AI.

The diagnosis and treatment of adrenal insufficiency in critical illness has been the subject of controversy for over 30 years. In 1977, Sibbald and colleagues reported that almost $20 \%$ of patients in septic shock have subnormal responses to ACTH administration. ${ }^{23}$ Since that time, however, the diagnosis (and even the existence) of CIRCI has been highly debated. Specifically, tumor necrosis factor (TNF) alfa and interleukin-1 (IL-1) have been implicated as inflammatory mediators in the reversible dysfunction of the HPA axis during critical illness. TNF- $\alpha$ likely impairs corticotropinreleasing hormone stimulated ACTH release, and a number of clinical studies have reported inappropriately low ACTH levels in patients with severe sepsis. ${ }^{24,25}$ The incidence of CIRCI varies widely from $10 \%$ to $71 \%$ depending of the definition used. ${ }^{26}$ However, making a clinical diagnosis is difficult in critically ill patients because systemic vascular resistance, cardiac output, and pulmonary capillary wedge pressure can be low, normal, or even high. ${ }^{27}$

While multiple tests have been developed to diagnose CIRCI, the most commonly used test is the ACTH stimulation test. In this setting, random cortisol levels are checked before a $250 \mu \mathrm{g}$ injection of cosyntropin and then 30-60 minutes later. The cortisol levels and response to cosyntropin may be interpreted to identify an insufficient adrenal response. Currently, either a random cortisol of less than 10 to $15 \mu \mathrm{g} / \mathrm{dL}$ or changes in cortisol of less than $9 \mu \mathrm{g} / \mathrm{dL}$ are the best tests for the diagnosis of adrenal insufficiency. Both values have an acceptably high specificity but low sensitivity. ${ }^{28}$ Several authors have demonstrated that nonsurvivors generally have higher baseline cortisol levels and a lower cortisol response to ACTH. ${ }^{26,29}$
However, free serum cortisol levels can be normal in critically ill patients who have low total serum cortisol; most likely as a result of a reduction in binding proteins during times of injury, stress and critical illness. ${ }^{30}$ Hamrahian and colleagues investigated total and free cortisol as well as cosyntropin stimulation responses in healthy volunteers and patients with sepsis. The authors found that while total cortisol levels were often consistent with adrenal insufficiency, free cortisol levels (not impacted by hypoproteinemia) demonstrated that these same patients had normal adrenal function. In patients with severe hypoproteinemia, adrenal insufficiency may be best defined by a baseline serum free cortisol concentration less than $2.0 \mu \mathrm{g} / \mathrm{dL}$ or ACTH-stimulated free cortisol concentrations less than $3.1 \mu \mathrm{g} / \mathrm{dL}$.

In the 1990's, several papers indicated a potential benefit of a brief course of corticosteroid therapy in critically ill patients (specifically those with vasopressor-dependent septic shock) who failed an ACTH stimulation test. ${ }^{8,31,32}$ However, in 2008, the results of the multicenter, multinational CORTICUS trial demonstrated that hydrocortisone cannot be recommended as general adjuvant therapy for vasopressor responsive septic shock. ${ }^{33}$ As well, the authors concluded that corticotropin testing could not be recommended to determine which patients should receive hydrocortisone therapy. In that trial, hydrocortisone did not improve survival or reverse shock, in patients with septic shock, either overall or in patients who did not respond to cosyntropin. It was noted however that in responders, the shock was reversed much faster. ${ }^{33}$

Of note is that the setting of critical illness can in, and of itself, greatly affect the cosyntropin test sensitivity in identifying adrenal insufficiency. The stress of critical illness and injury can greatly increase cortisol production while increasing glucocorticoid resistance. ${ }^{28}$ The changes in the stress response during critical illness combined with the resuscitation and management of these patients greatly disturbs serum protein levels, especially those of albumin and transcortin. With lower levels of these binding proteins, baseline cortisol and post-stimulation levels are generally lower than in similar states where albumin levels are normal. Complicating this, frequent intensive care unit (ICU) diagnoses such as sepsis and malnutrition can increase baseline levels and blunt the cortisol response to cosyntropin stimulation, respectively.

Further complicating the diagnosis of CIRCI in the ICU setting are the numerous commonly used agents that have been shown to interfere (in varying degrees) with cortisol levels and cosyntropin responsiveness. Several pharmacologic agents frequently used in the severely injured and 
critically ill populations have been shown to impair adrenal function and steroidogenesis. ${ }^{34-36}$ Propofol impairs adrenal steroidogenesis, while agents such as midazolam, morphine, and fentanyl have been shown to blunt the HPA axis, thereby interfering with corticosteroid metabolism. Etomidate is an imidazole derivative frequently used as an induction agent for rapid sequence intubation. In critically ill and injured patients, etomidate has gained increasing popularity for its rapid onset of action, cardiovascular stability, and limited respiratory depression. ${ }^{13}$ However, it is well established that adrenal suppression (via inhibition of 11-b hydroxylase), has brought the use of etomidate into question. ${ }^{13,37,38}$ In fact, among the major risk factors (coagulopathy, mechanical ventilation, hemorrhagic shock, vasopressor use, and septic shock) evaluated by Cotton and colleagues, etomidate use was the only modifiable risk factor identified. ${ }^{13}$

A single dose of etomidate has been demonstrated to inhibit cortisol production for up to 48 hours. ${ }^{39}$ Exposure to etomidate is a risk factor for the development of CIRCI in critically injured patients, and alternative drugs should be used when possible. ${ }^{13}$ In a reanalysis of a double blind clinical trial of 299 patients with septic shock randomized to receive placebo or corticosteroids, 77 (26\%) patients received etomidate..$^{40,41}$ Of these, $94 \%$ did not respond well to cosyntropin stimulation and the blockade of steroidogenesis lasted around 72 hours. More importantly, mortality was different between etomidate treated patients randomized to placebo $(76 \%)$ and those randomized to corticosteroids (55\%). This statistically significant absolute risk reduction of $21 \%$ translates into a number needed to treat of five patients.

Clearly steroids have a place in the ICU. However, their dosing and length of administration remain inconsistent. The use of extended course, stress-dose corticosteroids has been evaluated in ten randomized controlled clinical trials in critically ill patients with sepsis and acute respiratory distress syndrome (ARDS). This dosing strategy has been reported to be associated with a reduction in mortality, more rapid weaning of vasopressor agents, decreased ICU length of stay, and an increase in ventilator-free days in ARDS. ${ }^{28}$ Randomized controlled trials evaluating the outcomes of high-dose, short course corticosteroid treatment in ARDS and sepsis and have been unable to show improved outcomes, and there was a higher incidence of complications in the patients who received high-dose corticosteroids. ${ }^{42,43}$ Treatment with moderate-dose corticosteroids is recommended in patients with septic shock who have responded poorly to volume resuscitation and vasopressor agents. In patients with early severe ARDS and unresolving ARDS, treatment with moderate dose glucocorticoids should be considered before day 14 .

Although previous studies have suggested that the treatment of patients with septic shock should be based on the results of a cosyntropin stimulation test, the limitations of this test in diagnosing adrenal insufficiency in the setting of critical illness, combined with the benefit of corticosteroids in both responders and nonresponders suggest that this test should not be used to select patients likely to derive benefit from corticosteroids. ${ }^{44}$ In a recent critical appraisal of how to approach this patients population when relative or occult adrenal insufficiency is suspected, Marik recommends initiating treatment with stress-doses of corticosteroids in patients with vasopressor-dependent septic shock within 12 hours of presentation. He also recommends doing so without stimulation testing but rather based on clinical suspicion or random free cortisol levels.

\section{Conclusion}

Cosyntropin is a synthetic derivative of ACTH that is used in the evaluation and diagnosis of patients with adrenocortical insufficiency. Cosyntropin stimulation testing remains the cornerstone of diagnosing both primary and secondary adrenal insufficiency. While both the low-dose $(1 \mu \mathrm{g})$ and high-dose versions $(250 \mu \mathrm{g})$ have been shown to diagnose adrenal insufficiency, the $1 \mu \mathrm{g}$ dose has been shown to be more sensitive and specific. However, its poor specificity in secondary adrenal insufficiency limits its use in definitively ruling out this diagnosis. In the critically ill patient population presenting with CIRCI, based on currently available data, stimulation testing of adrenal function should not be routinely performed. Steroid therapy should be started presumptively in hemodynamically unstable patients while those who are stable may await random cortisol level results.

\section{Disclosure}

The authors report no conflicts of interest relevant to this research.

\section{References}

1. Oelkers W, Diederich S, Bahr V. Diagnosis of adrenal cortex insufficiency. Dtsch Med Wochenschr. 1994;119(15):555-559.

2. Kong MF, Jeffcoate W. Eighty-six cases of Addison's disease. Clin Endocrinol (Oxf). 1994;41(6):757-761.

3. Oelkers W. Adrenal insufficiency. $N$ Engl J Med. 1996;335(16): 1206-1212.

4. Topliss DJ, White EL, Stockigt JR. Significance of thyrotropin excess in untreated primary adrenal insufficiency. J Clin Endocrinol Metab. 1980;50(1):52-56

5. Muls E, Bouillon R, Boelaert J, et al. Etiology of hypercalcemia in a patient with Addison's disease. Calcif Tissue Int. 1982;34(6):523-526. 
6. Cooper MS, Stewart PM. Corticosteroid insufficiency in acutely ill patients. N Engl J Med. 2003;348(8):727-734.

7. Barton RN, Stoner HB, Watson SM. Relationships among plasma cortisol, adrenocorticotrophin, and severity of injury in recently injured patients. J Trauma. 1987;27(4):384-392.

8. Briegel J, Forst H, Haller M, et al. Stress doses of hydrocortisone reverse hyperdynamic septic shock: a prospective, randomized, double-blind, single-center study. Crit Care Med. 1999;27(4):723-732.

9. Cohan P, Wang C, McArthur DL, et al. Acute secondary adrenal insufficiency after traumatic brain injury: a prospective study. Crit Care Med. 2005;33(10):2358-2366.

10. Nieboer P, van der Werf TS, Beentjes JA, Tulleken JE, Zijlstra JG, Ligtenberg JJ. Catecholamine dependency in a polytrauma patient: relative adrenal insufficiency? Intensive Care Med. 2000;26(1):125-127.

11. Rivers EP, Gaspari M, Saad GA, et al. Adrenal insufficiency in high-risk surgical ICU patients. Chest. 2001;119(3):889-896.

12. Baldwin WA, Allo M. Occult hypoadrenalism in critically ill patients. Arch Surg. 1993;128(6):673-676.

13. Cotton BA, Guillamondegui OD, Fleming SB, et al. Increased risk of adrenal insufficiency following etomidate exposure in critically injured patients. Arch Surg. 2008;143(1):62-67.

14. Offner PJ, Moore EE, Ciesla D. The adrenal response after severe trauma. Am J Surg. 2002;184(6):649-653; discussion 653-644.

15. Dunn JF, Nisula BC, Rodbard D. Transport of steroid hormones: binding of 21 endogenous steroids to both testosterone-binding globulin and corticosteroid-binding globulin in human plasma. $J$ Clin Endocrinol Metab. 1981;53(1):58-68.

16. Mueller UW, Potter JM. Binding of cortisol to human albumin and serum: the effect of protein concentration. Biochem Pharmacol. 1981;30(7):727-733.

17. Thomas JP, el-Shaboury AH. Aldosterone secretion in steroid-treated patients with adrenal suppression. Lancet. 1971;1(7700):623-625.

18. Cortrosyn package insert. Rancho Cucamonga, CA: Amphastar Pharmaceuticals, Inc.; May 2003.

19. Wheeler MH, Harris DA. Diagnosis and management of primary aldosteronism. World J Surg. 2003;27(6):627-631.

20. Dorin RI, Qualls CR, Crapo LM. Diagnosis of adrenal insufficiency. Ann Intern Med. 2003;139(3):194-204.

21. Asare K. Diagnosis and treatment of adrenal insufficiency in the critically ill patient. Pharmacotherapy. 2007;27(11):1512-1528.

22. Arlt W, Allolio B. Adrenal insufficiency. Lancet. 2003;361(9372): 1881-1893.

23. Sibbald WJ, Short A, Cohen MP, Wilson RF. Variations in adrenocortical responsiveness during severe bacterial infections. Unrecognized adrenocortical insufficiency in severe bacterial infections. Ann Surg. 1977;186(1):29-33.

24. Gaillard RC, Turnill D, Sappino P, Muller AF. Tumor necrosis factor alpha inhibits the hormonal response of the pituitary gland to hypothalamic releasing factors. Endocrinology. 1990;127(1): 101-106.

25. Soni A, Pepper GM, Wyrwinski PM, et al. Adrenal insufficiency occurring during septic shock: incidence, outcome, and relationship to peripheral cytokine levels. Am J Med. 1995;98(3):266-271.
26. Lipiner-Friedman D, Sprung CL, Laterre PF, et al. Adrenal function in sepsis: the retrospective Corticus cohort study. Crit Care Med. 2007;35(4):1012-1018.

27. Zaloga GP, Marik P. Hypothalamic-pituitary-adrenal insufficiency. Crit Care Clin. 2001;17(1):25-41.

28. Marik PE. Critical illness-related corticosteroid insufficiency. Chest. 2009;135(1):181-193.

29. Annane D, Sebille V, Troche G, Raphael JC, Gajdos P, Bellissant E. A 3-level prognostic classification in septic shock based on cortisol levels and cortisol response to corticotropin. JAMA. 2000;283(8): $1038-1045$

30. Hamrahian AH, Oseni TS, Arafah BM. Measurements of serum free cortisol in critically ill patients. $N$ Engl J Med. 2004;350(16):1629-1638.

31. Bollaert PE, Charpentier C, Levy B, Debouverie M, Audibert G, Larcan A. Reversal of late septic shock with supraphysiologic doses of hydrocortisone. Crit Care Med. 1998;26(4):645-650.

32. Annane D, Bellissant E. Prognostic value of cortisol response in septic shock. JAMA. 2000;284(3):308-309.

33. Sprung CL, Annane D, Keh D, et al. Hydrocortisone therapy for patients with septic shock. N Engl J Med. 2008;358(2):111-124.

34. Aitkenhead AR, Pepperman ML, Willatts SM, et al. Comparison of propofol and midazolam for sedation in critically ill patients. Lancet. 1989;2(8665):704-709.

35. Brooks SM, Werk EE, Ackerman SJ, Sullivan I, Thrasher K. Adverse effects of phenobarbital on corticosteroid metabolism in patients with bronchial asthma. N Engl J Med. 1972;286(21):1125-1128.

36. Newman LH, McDonald JC, Wallace PG, Ledingham IM. Propofol infusion for sedation in intensive care. Anaesthesia. 1987;42(9): 929-937.

37. Jackson WL Jr. Should we use etomidate as an induction agent for endotracheal intubation in patients with septic shock?: a critical appraisal. Chest. 2005;127(3):1031-1038.

38. Roberts RG, Redman JW. Etomidate, adrenal dysfunction and critical care. Anaesthesia. 2002;57(4):413.

39. Vinclair M, Broux C, Faure P, et al. Duration of adrenal inhibition following a single dose of etomidate in critically ill patients. Intensive Care Med. 2008;34(4):714-719.

40. Annane D. ICU physicians should abandon the use of etomidate! Intensive Care Med. 2005;31(3):325-326.

41. Annane D, Sebille V, Charpentier C, et al. Effect of treatment with low doses of hydrocortisone and fludrocortisone on mortality in patients with septic shock. JAMA. 2002;288(7):862-871.

42. Annane D, Bellissant E, Bollaert PE, Briegel J, Keh D, Kupfer Y. Corticosteroids for severe sepsis and septic shock: a systematic review and meta-analysis. BMJ. 2004;329(7464):480.

43. Minneci PC, Deans KJ, Banks SM, Eichacker PQ, Natanson C. Meta-analysis: the effect of steroids on survival and shock during sepsis depends on the dose. Ann Intern Med. 2004;141(1):47-56.

44. Marik PE, Pastores SM, Annane D, et al. Recommendations for the diagnosis and management of corticosteroid insufficiency in critically ill adult patients: consensus statements from an international task force by the American College of Critical Care Medicine. Crit Care Med. 2008;36(6):1937-1949.
Clinical Pharmacology: Advances and Applications

\section{Publish your work in this journal}

Clinical Pharmacology: Advances and Applications is an international, peer-reviewed, open access journal publishing original research, reports, reviews and commentaries on all areas of drug experience in humans. The manuscript management system is completely online and includes a very quick and fair peer-review system, which is all easy to use.

\section{Dovepress}

Visit http://www.dovepress.com/testimonials.php to read real quotes from published authors. 